\title{
Origin of the magnetic anomaly and tunneling effect of europium on the ferromagnetic ordering in $\mathrm{Eu}_{8-\mathrm{x}} \mathrm{Sr}_{\mathrm{x}} \mathrm{Ga}_{16} \mathrm{Ge}_{30}(x=0,4)$ type-I clathrates
}

\author{
Manh-Huong Phan, ${ }^{1}$ Victorino Franco, ${ }^{1,2}$ Anurag Chaturvedi, ${ }^{1}$ Stevce Stefanoski, ${ }^{1}$ George S. Nolas, ${ }^{1, *}$ and \\ Hariharan Srikanth ${ }^{1, \dagger}$ \\ ${ }^{1}$ Department of Physics, University of South Florida, Tampa, Florida 33620, USA \\ ${ }^{2}$ Dpto. Física de la Materia Condensada, ICMSE-CSIC, Universidad de Sevilla, P.O. Box 1065, 41080 Sevilla, Spain
}

(Received 17 September 2010; revised manuscript received 6 April 2011; published 11 August 2011)

\begin{abstract}
Systematic dc magnetization studies using the Banerjee criterion, Kouvel-Fisher, and magnetocaloric effect methods provide physical insights into the origin of the magnetic anomaly and the tunneling effect of europium on the ferromagnetic ordering in $\mathrm{Eu}_{8} \mathrm{Ga}_{16} \mathrm{Ge}_{30}$ type-I clathrates. We show that $\mathrm{Eu}_{8} \mathrm{Ga}_{16} \mathrm{Ge}_{30}$ undergoes a second-order magnetic transition (SOMT) at $T_{C} \sim 35 \mathrm{~K}$, resulting from the magnetic interaction between the $\mathrm{Eu}^{2+}$ ions at the Eu2 sites, followed by a secondary magnetic transition at $T_{L} \sim 10 \mathrm{~K}$ (indicated as a magnetic anomaly in previous studies), as a result of the magnetic interaction between the $\mathrm{Eu}^{2+}$ ions at the Eu1 and Eu2 sites. The critical exponent $\beta=0.388$ is close to that predicted from the three-dimensional Heisenberg model $(\beta=0.365)$, while the critical exponent $\gamma=0.956$ is close to that predicted from the mean-field model $(\gamma=1)$. The substitution of $\mathrm{Sr}^{2+}$ for $\mathrm{Eu}^{2+}$ retains the SOMT but largely reduces the transition temperatures $\left(T_{C} \sim 15 \mathrm{~K}\right.$ and $\left.T_{L} \sim 5 \mathrm{~K}\right)$, with the critical exponents $\beta=0.521$ and $\gamma=0.917$ close to those predicted from the mean-field model $(\beta=0.5$ and $\gamma=1$ ). These results point to the important fact that the tunneling of $\mathrm{Eu}^{2+}$ between the four equivalent sites in the tetrakaidecahedral cage tends to prevent the occurrence of a long-range ferromagnetic ordering in the type-I clathrate materials.
\end{abstract}

DOI: 10.1103/PhysRevB.84.054436

PACS number(s): 75.30.Sg

\section{INTRODUCTION}

In recent years, semiconductors with the clathrate-hydrate crystal structure have been the subject of intense research due to their potential for thermoelectric applications. ${ }^{1-3}$ In these materials "guest" atoms of one or more species reside inside "host" framework polyhedra formed by other species. ${ }^{1}$ Incorporating rare-earth elements with large magnetic moments as "guest" atoms into an adequate semiconducting host framework has made these materials also interesting for thermomagnetic applications. ${ }^{4-8}$ Among the existing clathrates, the composition $\mathrm{Eu}_{8} \mathrm{Ga}_{16} \mathrm{Ge}_{30}$ is an example in which the sites for the guest atoms are fully occupied by Eu. ${ }^{4,5}$ The $\mathrm{Eu}_{8} \mathrm{Ga}_{16} \mathrm{Ge}_{30}$ composition can form in two completely different clathrate structure types. ${ }^{5}$ In the type-I $(P m \overline{3} n)$ clathrate crystal structure, Eu resides inside two different polyhedra: two inside dodecahedra at Eu1 ( $2 a$ crystallographic site) and six inside tetrakaidecahedra at Eu2 (6d crystallographic site) per unit cell. In the type-VIII $(I \overline{4} 3 m)$ clathrate structure, eight distorted pentagonal dodecahedra containing 23 vertices surround the $\mathrm{Eu}^{2+}$ ions. $^{5}$ The ferromagnetism of the clathrates is attributed to the presence of $\mathrm{Eu}^{2+}$ ions with a large saturation moment $\left(7 \mu_{B} / \mathrm{Eu}\right)$. The large separation distance between neighboring $\mathrm{Eu}^{2+}$ ions $(>5.23 \AA)$ excludes the direct exchange interaction between the localized $4 f$ moments and suggests the long-range Ruderman-Kittel-Kasuya-Yoshida (RKKY) interaction via the charge carriers as the key mechanism for the occurrence of the ferromagnetism in these materials. ${ }^{4-8}$ Since the shortest distance between $\mathrm{Eu}^{2+}$ ions in the type-I clathrate $(\sim 5.23 \AA)$ is smaller than that of the type-VIII clathrate $(\sim 5.562 \AA)$, the former possesses a higher Curie temperature $\left(T_{C} \sim 35 \mathrm{~K}\right)$ compared with that of the latter $\left(T_{C} \sim 13 \mathrm{~K}\right)^{5,7}$ Using the standard formulation for the exchange Hamiltonian and the carrier concentrations at $T_{C}$ determined from the Hall constants, Paschen et al. ${ }^{5}$ have argued that ferromagnetism should exist for Eu-Eu distances under $6.5 \AA$ for the type-I clathrate and $10 \AA$ for the type-VIII clathrate. Nevertheless, the difference in the Curie temperature between these two clathrates has recently been re-examined and attributed to the different effective masses of the charge carriers. ${ }^{9,10}$ Our recent studies on the $\mathrm{Eu}_{8} \mathrm{Ga}_{16} \mathrm{Ge}_{30}$ type-VIII clathrate have revealed the nature of a second-order, paramagnetic to ferromagnetic (PM-FM) transition at $T_{C} \sim 13 \mathrm{~K}$, with long-range ferromagnetic ordering. ${ }^{7}$ However, the case may be different for the $\mathrm{Eu}_{8} \mathrm{Ga}_{16} \mathrm{Ge}_{30}$ type-I clathrate. ${ }^{4-6,8}$ In this material, the shortest distances for Eu2-Eu2, Eu2-Eu1, and Eu1-Eu1 are $5.23 \AA$, $5.62 \AA$, and $10.7 \AA$, respectively. ${ }^{5}$ The Eu-Eu distance in the [111] direction is $9.27 \AA$. Since the ferromagnetism only exists within Eu-Eu distances of $6.5 \AA,{ }^{5}$ the magnetic interactions at the Eu2-Eu2 and Eu1-Eu2 distances are expected to contribute to the ferromagnetism of the material. The ferromagnetism is dominated by the magnetic interaction between the $\mathrm{Eu}^{2+}$ ions at the Eu2 sites, because the Eu2-Eu2 distance $(5.23 \AA)$ is shorter than the Eu1-Eu2 distance $(5.62 \AA)$ and more $\mathrm{Eu}^{2+}$ ions are located at the Eu2 sites than at the Eu1 sites per unit cell. However, the magnetic interaction between the $\mathrm{Eu}^{2+}$ ions at the Eu1 and Eu2 sites is also expected to be significant at low temperature, which may result in a secondary ferromagnetic ordering. This is likely an important clue in assessing the physical origin of the broad hump observed in $\rho(T)$ and $C_{P}(T)$ at around $10 \mathrm{~K}$ (henceforth $T_{L}$ ) in the $\mathrm{Eu}_{8} \mathrm{Ga}_{16} \mathrm{Ge}_{30}$ type-I clathrate, which remained unexplained satisfactorily previously ${ }^{5,10}$ Furthermore, it has been noted that $\mathrm{Eu}^{2+}$ tunnels between four equivalent sites in the tetrakaidecahedral cages (at the Eu2 sites) in the $\mathrm{Eu}_{8} \mathrm{Ga}_{16} \mathrm{Ge}_{30}$ type-I clathrate. ${ }^{11}$ This effect was observed in the ferromagnetic phase below $T_{C} \sim 35 \mathrm{~K}^{12,13}$ and has recently been argued to account for the formation of the modulated ferromagnetic structure in the type-I clathrate. ${ }^{14}$ However, there are two emerging questions: 
Why does the jump in $C_{p}$ at $\mathrm{T}_{C}$ significantly differ from the value expected for a uniform ferromagnet with $S=7 / 2$ from the mean-field theory $?^{4,5,14}$ What is the universality class that governs the PM-FM transition in the $\mathrm{Eu}_{8} \mathrm{Ga}_{16} \mathrm{Ge}_{30}$ type-I clathrate? In this context, we believe that a clear understanding of the physical origin of the magnetic anomaly, the nature of the PM-FM transition, and the influence of tunneling of $\mathrm{Eu}^{2+}$ between the four equivalent sites in the tetrakaidecahedral cage on the critical behavior near $T_{C}$ in the type-I clathrate materials is of importance.

To address these important and as yet unresolved issues, we have conducted a comprehensive study of the ferromagnetic phase transition and critical exponents in $\mathrm{Eu}_{8} \mathrm{Ga}_{16} \mathrm{Ge}_{30}$ and $\mathrm{Eu}_{4} \mathrm{Sr}_{4} \mathrm{Ga}_{16} \mathrm{Ge}_{30}$ type-I clathrates. The critical exponents associated with this transition have been determined from dc magnetization data using the Kouvel-Fisher (K-F) and the magnetocaloric effect (MCE) methods. Our results reveal that the magnetic transition is of second-order type in both cases. We show that in $\mathrm{Eu}_{8} \mathrm{Ga}_{16} \mathrm{Ge}_{30}$ in addition to the PM-FM transition at $T_{C} \sim 35 \mathrm{~K}$, a secondary magnetic transition is present at $T_{L} \sim 10 \mathrm{~K}$. We believe that the transition at $T_{C}$ results from the magnetic interaction between the $\mathrm{Eu}^{2+}$ ions at the $\mathrm{Eu} 2$ sites, while the transition at $T_{L}$ originates from the magnetic interaction between the $\mathrm{Eu}^{2+}$ ions at the Eu1 $(2 a)$ and Eu2 $(6 d)$ sites. The finding of the secondary magnetic transition at $T_{L} \sim 10 \mathrm{~K}$ can explain the occurrence of the broad hump in $\rho(T)$ and $C_{P}(\mathrm{~T})$ at approximately $10 \mathrm{~K}$. Following the usual notation, the critical exponent $\beta$ describes the temperature dependence of the spontaneous magnetization $\left.\left(M_{0} \propto\left(T-T_{C}\right)^{\beta}\right)\right)$, the exponent $\gamma$ describes that of the zero-field paramagnetic susceptibility $\chi_{0}^{-1} \propto\left(T-T_{C}\right)^{\gamma}$, and $n\left(T_{C}\right)$ describes the field dependence of the magnetic entropy change at the critical temperature $\left(\Delta S_{M}\left(T=T_{C}\right) \propto H^{n\left(T_{C}\right)}\right)$. For $\mathrm{Eu}_{8} \mathrm{Ga}_{16} \mathrm{Ge}_{30} \beta=0.388$ is close to that predicted from the three-dimensional (3D) Heisenberg model $(\beta=0.365)$, while $\gamma=0.956$ is close to that predicted from the mean-field model $(\gamma=1) \cdot n\left(T_{C}\right)=0.545$ significantly deviates from that predicted by the mean-field model $\left(n\left(T_{C}\right)=2 / 3\right)$. The $\mathrm{Sr}$ doping largely reduces the transition temperatures $\left(T_{C} \sim 15 \mathrm{~K}\right.$ and $\left.T_{L} \sim 5 \mathrm{~K}\right)$, with the critical exponents $\beta=0.521$, $\gamma=0.917$, and $n\left(T_{C}\right)=0.667$ close to those predicted by the mean-field model $\left(\beta=0.5, \gamma=1\right.$ and $\left.n\left(T_{C}\right)=2 / 3\right)$. These findings suggest the influence of tunneling of $\mathrm{Eu}^{2+}$ between the four equivalent sites in the tetrakaidecahedral cage (at the Eu2 sites) on the ferromagnetic correlation in the type-I clathrate and allow us to address why the magnitude of the jump in $C_{p}$ at $T_{C}$ significantly differs from the value expected for a uniform ferromagnet with $S=7 / 2$ from the mean-field theory. ${ }^{4,5,14}$

\section{EXPERIMENT}

Polycrystalline $\mathrm{Eu}_{8} \mathrm{Ga}_{16} \mathrm{Ge}_{30}$ with the type-I crystal structure was synthesized by reacting the high-purity elements in stoichiometric ratios inside a boron nitride $(\mathrm{BN})$ crucible, which was enclosed in a nitrogen atmosphere, inside a sealed quartz ampoule. The specimen was placed in an induction furnace at $1000{ }^{\circ} \mathrm{C}$ for 10 minutes followed by a rapid water quench. The $\mathrm{Eu}_{4} \mathrm{Sr}_{4} \mathrm{Ga}_{16} \mathrm{Ge}_{30}$ specimen was prepared by reacting the high-purity elements in a $\mathrm{BN}$ crucible inside a nitrogen-filled sealed quartz tube at $950{ }^{\circ} \mathrm{C}$

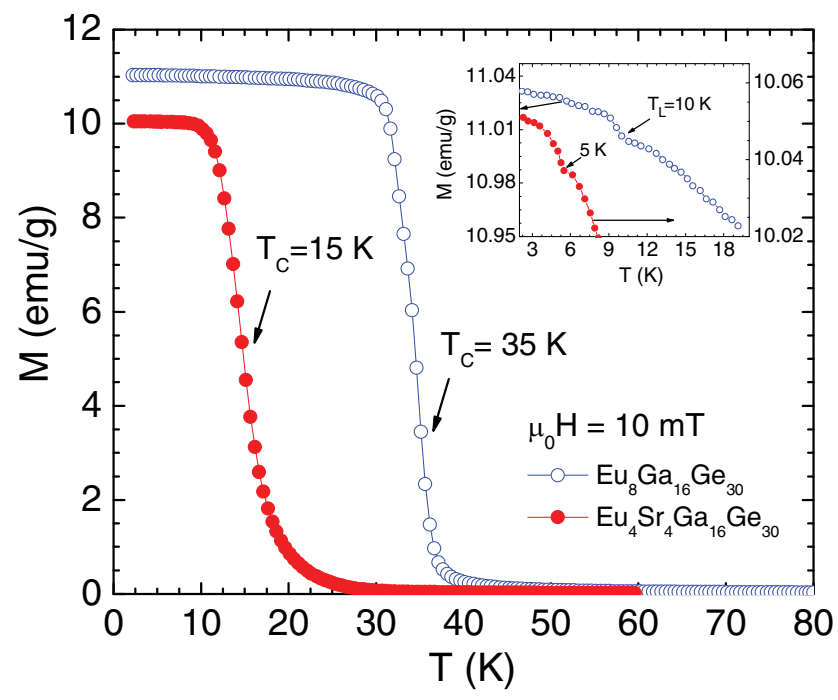

FIG. 1. (Color online) Temperature dependence of magnetization taken at a field of $10 \mathrm{mT}$ for $\mathrm{Eu}_{8} \mathrm{Ga}_{16} \mathrm{Ge}_{30}$ and $\mathrm{Eu}_{4} \mathrm{Sr}_{4} \mathrm{Ga}_{16} \mathrm{Ge}_{30}$. The inset shows the enlarged M-T curve for $\mathrm{Eu}_{8} \mathrm{Ga}_{16} \mathrm{Ge}_{30}$.

for 3 days, followed by slow cooling to $700{ }^{\circ} \mathrm{C}$. After 3 days at $700{ }^{\circ} \mathrm{C}$, the product was air quenched..$^{6,15,16} \mathrm{X}$-ray diffraction (XRD) and energy dispersive spectroscopy (EDS) analyses revealed the presence of the type-I clathrate phase only, with no oxide impurities, and homogenous compositions within the polycrystalline grains. ${ }^{17}$ Refinement of synchrotron powder diffraction patterns revealed for a stoichiometry of $\mathrm{Eu}_{3.47(3)} \mathrm{Sr}_{4.53(3)} \mathrm{Ga}_{14.48(13)} \mathrm{Ge}_{31.52(13)}$ with a $76 \%$ preferential europium occupation of the Eu1 site. ${ }^{16}$ Magnetic and magnetocaloric measurements were conducted using a commercial Physical Property Measurement System from Quantum Design over a temperature range of $2-60 \mathrm{~K}$ at applied fields up to $3 \mathrm{~T}$.

\section{RESULTS AND DISCUSSION}

Figure 1 shows the temperature dependence of the fieldcooled (FC) magnetization taken at a low applied field of $10 \mathrm{mT}$ for $\mathrm{Eu}_{8} \mathrm{Ga}_{16} \mathrm{Ge}_{30}$ and $\mathrm{Eu}_{4} \mathrm{Sr}_{4} \mathrm{Ga}_{16} \mathrm{Ge}_{30}$. The PM-FM transitions in both specimens are noted. The Curie temperatures $\left(T_{\mathrm{C}}\right)$, which are determined from the minimum in $d M / d T$ across the transitions, are $35 \mathrm{~K}$ and $15 \mathrm{~K}$ for $\mathrm{Eu}_{8} \mathrm{Ga}_{16} \mathrm{Ge}_{30}$ and $\mathrm{Eu}_{4} \mathrm{Sr}_{4} \mathrm{Ga}_{16} \mathrm{Ge}_{30}$, respectively. A closer examination reveals a sharp increase in the magnetization at $\sim 10 \mathrm{~K}$ for $\mathrm{Eu}_{8} \mathrm{Ga}_{16} \mathrm{Ge}_{30}$ and at $\sim 5 \mathrm{~K}$ for $\mathrm{Eu}_{4} \mathrm{Sr}_{4} \mathrm{Ga}_{16} \mathrm{Ge}_{30}$ (see inset of Fig. 1). This increase in the FC magnetization at $T_{L}$ is a clear indication of a secondary magnetic transition. We believe this transition originates from the magnetic interactions between the $\mathrm{Eu}^{2+}$ ions at the Eu1 and Eu2 sites (determined by the Eu1-Eu2 distance). Our previous studies have revealed that $\mathrm{Sr}^{2+}$ preferentially replaces $\mathrm{Eu}^{2+}$ on the $\mathrm{Eu} 2$ sites in $\mathrm{Eu}_{4} \mathrm{Sr}_{4} \mathrm{Ga}_{16} \mathrm{Ge}_{30}$. This substitution increases the $\mathrm{Eu}$-Eu distance to at least $\sim 10 \mathrm{~A}^{\circ}{ }^{6}$ Therefore, a strong decrease in the $T_{C}, T_{L}$, and saturation magnetization $\left(M_{S}\right)$ is expected in the $\mathrm{Sr}$ containing specimen.

To determine the type of the PM-FM transition in $\mathrm{Eu}_{8} \mathrm{Ga}_{16} \mathrm{Ge}_{30}$ and $\mathrm{Eu}_{4} \mathrm{Sr}_{4} \mathrm{Ga}_{16} \mathrm{Ge}_{30}$, we have analyzed $H / M$ vs $M^{2}$ curves (which were converted from the isothermal $M-H$ data), using the so-called Banerjee criterion. ${ }^{18}$ The results are 

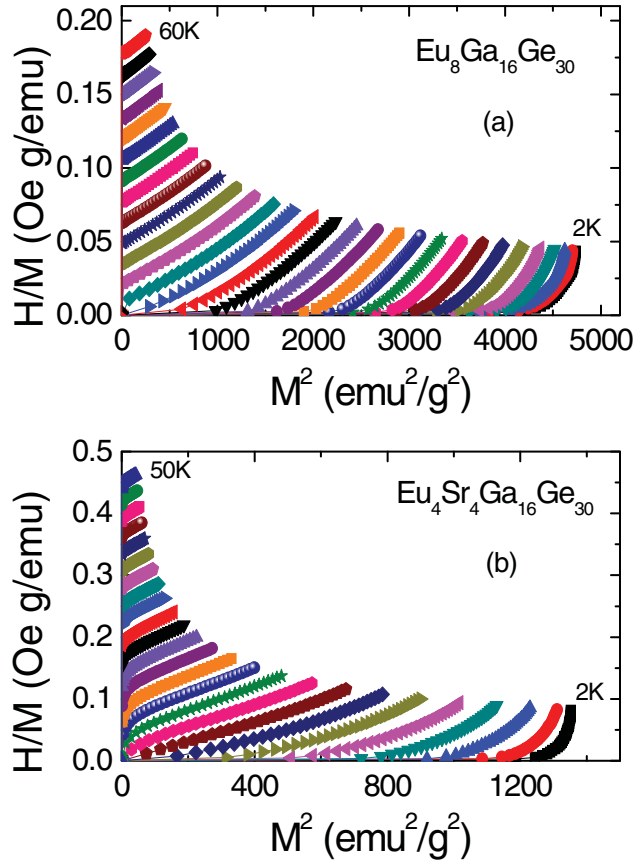

FIG. 2. (Color online) The $H / M$ vs $M^{2}$ plots at representative temperatures around $T_{C}$ for $\mathrm{Eu}_{8} \mathrm{Ga}_{16} \mathrm{Ge}_{30}$ and $\mathrm{Eu}_{4} \mathrm{Sr}_{4} \mathrm{Ga}_{16} \mathrm{Ge}_{30}$.

presented in Fig. 2. According to this criterion, the magnetic transition is of second order if all the $H / M$ vs $M^{2}$ curves have a positive slope. ${ }^{19}$ On the other hand, if some of the $H / M$ vs $M^{2}$ curves show a negative slope at some point, the transition is considered to be of first order. ${ }^{19,20}$ In the present case, the positive slopes of the $H / M$ vs $M^{2}$ curves of $\mathrm{Eu}_{8} \mathrm{Ga}_{16} \mathrm{Ge}_{30}$ and $\mathrm{Eu}_{4} \mathrm{Sr}_{4} \mathrm{Ga}_{16} \mathrm{Ge}_{30}$ clearly indicate that these are second-order magnetic transition (SOMT) materials. This result is consistent with the reported $\lambda$-shape of the specific heat $C_{p}(T)$ curve in the $\mathrm{Eu}_{8} \mathrm{Ga}_{16} \mathrm{Ge}_{30}$ type-I clathrate. ${ }^{4,5}$

Since the $\mathrm{Eu}_{8} \mathrm{Ga}_{16} \mathrm{Ge}_{30}$ and $\mathrm{Eu}_{4} \mathrm{Sr}_{4} \mathrm{Ga}_{16} \mathrm{Ge}_{30}$ specimens exhibit SOMT, we have used the K-F method ${ }^{21}$ to precisely determine the critical exponents near their PM-FM transition temperatures. This method consists of an iterative procedure, which starts by constructing the Arrott-Noakes (A-N) plot (i.e., the plot of $M^{2.5}$ vs $\left.(H / M)^{0.75}\right)$. From this plot, the values for the spontaneous magnetization $M_{0}(T)$ are computed from the intercepts of various isothermal magnetization vs field curves on the ordinate of the plot (for temperatures below $T_{C}$ ). The intercept on the abscissa (for temperatures above $\mathrm{T}_{\mathrm{C}}$ ), allows us to calculate the zero-field paramagnetic susceptibility $\chi_{0}(T)$.

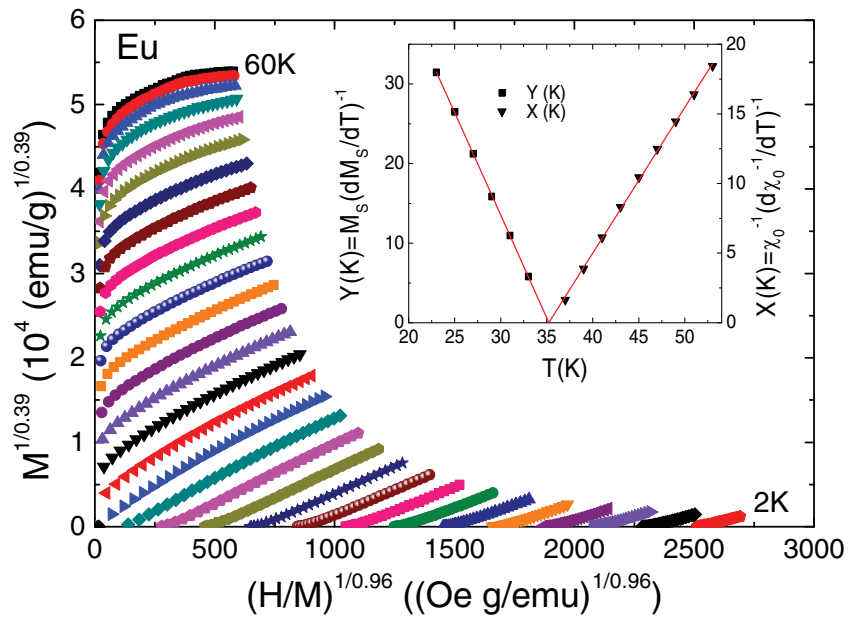

FIG. 3. (Color online) Modified Arrott plot isotherms with 2-K temperature interval for $\mathrm{Eu}_{8} \mathrm{Ga}_{16} \mathrm{Ge}_{30}$. The inset shows the K-F construction for determining the critical exponents and the Curie temperature; the solid lines are fits to Eqs. (1) and (2), respectively.

Once the $M_{0}(T)$ and $\chi_{0}(T)$ curves have been constructed, two additional parameter data sets, $X(T)$ and $Y(T)$, may be determined:

$$
\begin{gathered}
X(T)=\chi_{0}^{-1}\left(d \chi_{0}^{-1} / d T\right)^{-1}=\left(T-T_{C}\right) / \gamma \\
Y(T)=M_{0}\left(d M_{0} / d T\right)^{-1}=\left(T-T_{C}\right) / \beta
\end{gathered}
$$

The rationale for constructing these quantities is that the spontaneous magnetization should scale with temperature as $M_{0} \propto\left(T-T_{C}\right)^{\beta}$ and with zero-field paramagnetic susceptibility as $\chi_{0}^{-1} \propto\left(T-T_{C}\right)^{\gamma}$. Therefore, in the critical region, both $X(T)$ and $Y(T)$ should be linear. The slopes give the values of the critical exponents and the intercepts on the temperature axis correspond to the Curie temperature. The values of the critical exponents are refined by using an iterative method, i.e., once Eqs. (1) and (2) produce the values of the critical exponents, a generalized A-N plot $\left(M^{1 / \beta}\right.$ vs $\left.(H / M)^{1 / \gamma}\right)$ is constructed and used to calculate new $M_{0}(T)$ and $\chi_{0}(T)$ curves, which are subsequently input into Eqs. (1) and (2), resulting in new values for $\beta$ and $\gamma$. The procedure terminates when the desired convergence of the parameters is achieved. $T_{C}$ is obtained as the intercept on the abscissa of both the $X$ and $Y$ lines.

As an example, Fig. 3 shows the A-N plots of $\mathrm{Eu}_{8} \mathrm{Ga}_{16} \mathrm{Ge}_{30}$ with optimized critical exponents $(\beta$ and $\gamma$ ) obtained from the K-F method. The inset of Fig. 3 shows the K-F plot

TABLE I. Table 1. Comparison of the values of the critical exponents of $\mathrm{Eu}_{8} \mathrm{Ga}_{16} \mathrm{Ge}_{30}$ and $\mathrm{Eu}_{4} \mathrm{Sr}_{4} \mathrm{Ga}_{16} \mathrm{Ge}_{30}$ type-I clathrates with those of

\begin{tabular}{|c|c|c|c|c|c|c|c|}
\hline Material & $\mathrm{T}_{\mathrm{C}}(\mathrm{K})$ & $\beta^{*}$ & $\gamma^{*}$ & $\delta$ & $\mathrm{n}(\mathrm{K}-\mathrm{F})$ & $\mathrm{n}(\mathrm{MCE})$ & Ref. \\
\hline $\mathrm{Eu}_{8} \mathrm{Ga}_{16} \mathrm{Ge}_{30}$ (type-I) & 35.2 & 0.388 & 0.956 & 3.474 & 0.545 & 0.562 & This work \\
\hline $\mathrm{Eu}_{4} \mathrm{Sr}_{4} \mathrm{Ga}_{16} \mathrm{Ge}_{30}$ (type-I) & 17.7 & 0.521 & 0.917 & 2.760 & 0.667 & 0.675 & This work \\
\hline $\mathrm{Eu}_{8} \mathrm{Ga}_{16} \mathrm{Ge}_{30}$ (type-VIII) & 13 & 0.470 & 0.964 & 3.014 & - & - & 7 \\
\hline Mean-field model & & 0.5 & 1.0 & 3.0 & 0.66 & & 36 \\
\hline 3D Heisenberg model & & 0.365 & 1.336 & 4.80 & & & 36 \\
\hline 3D Ising model & & 0.325 & 1.241 & 4.82 & & & 36 \\
\hline
\end{tabular}
theoretical models and of the $\mathrm{Eu}_{8} \mathrm{Ga}_{16} \mathrm{Ge}_{30}$ type-VIII clathrate from our previous study. ${ }^{7}$

*The errors for calculating $\beta$ and $\gamma$ are less than 0.05 . 

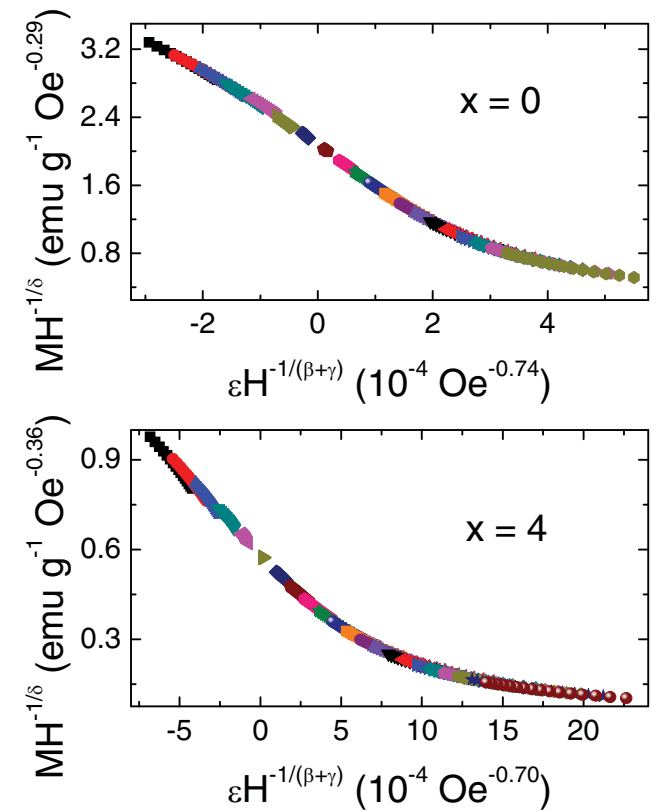

FIG. 4. (Color online) Normalized isotherms of $\mathrm{Eu}_{8} \mathrm{Ga}_{16} \mathrm{Ge}_{30}$ and $\mathrm{Eu}_{4} \mathrm{Sr}_{4} \mathrm{Ga}_{16} \mathrm{Ge}_{30}$ below and above the Curie temperature $\left(T_{C}\right)$ using the values of $\beta$ and $\gamma$ determined by the K-F method.

for this specimen. The fit parameters of both specimens are summarized in Table I. The best fit yields the values of $T_{C}=$ $35.2 \mathrm{~K}, \beta=0.388$, and $\gamma=0.956$ for $\mathrm{Eu}_{8} \mathrm{Ga}_{16} \mathrm{Ge}_{30}$ and $T_{C}$ $=7.7 \mathrm{~K}, \beta=0.521$, and $\gamma=0.917$ for $\mathrm{Eu}_{4} \mathrm{Sr}_{4} \mathrm{Ga}_{16} \mathrm{Ge}_{30}$. Using the Widom scaling relationship, ${ }^{22} \beta+\gamma=\beta \delta$, the critical exponent $\delta$ is determined to be 3.474 and 2.760 for $\mathrm{Eu}_{8} \mathrm{Ga}_{16} \mathrm{Ge}_{30}$ and $\mathrm{Eu}_{4} \mathrm{Sr}_{4} \mathrm{Ga}_{16} \mathrm{Ge}_{30}$, respectively. This relationship has been tested by plotting $M\left(T=T_{C}\right)$ vs $H^{\beta /(\beta+\gamma)}=H^{1 / \delta}$ and checking the linearity of the curve (not shown).

The reliability of the obtained exponents and Curie temperatures of $\mathrm{Eu}_{8} \mathrm{Ga}_{16} \mathrm{Ge}_{30}$ and $\mathrm{Eu}_{4} \mathrm{Sr}_{4} \mathrm{Ga}_{16} \mathrm{Ge}_{30}$ can also be ascertained by checking the scaling of the magnetization curves. It has been shown that for magnetic systems, the scaling equation of state takes the form ${ }^{23}$

$$
\frac{H}{M^{\delta}}=h\left(\frac{\varepsilon}{M^{1 / \beta}}\right),
$$

where $\varepsilon=\left(T-T_{C}\right) / T_{C}$ is the reduced temperature, $T_{C}$ is the Curie temperature, $h(x)$ is a scaling function, and $\beta$ and $\gamma$ are critical exponents, which characterize the magnetization behavior along the coexistence $(H=0, \varepsilon<0)$ and the critical isotherm $(\varepsilon=0)$, respectively. Therefore, according to Eq. (3), if the appropriate values for $\beta, \gamma$, and $T_{C}$ are used, the plot of $M / H^{1 / \delta}$ vs $\varepsilon / H^{1 / \Delta}$ should correspond to a universal curve onto which all experimental data points collapse. Using the values of $\beta, \gamma$, and $T_{C}$ obtained from the K-F method, the scaled data are plotted in Fig. 4 for both specimens. The excellent overlap of the experimental data points clearly indicates that the obtained values of $\beta, \gamma$, and $T_{C}$ for these specimens are in agreement with the scaling hypothesis.

To get additional information about the magnetic ground states and critical behavior near the PM-FM transition in $\mathrm{Eu}_{8} \mathrm{Ga}_{16} \mathrm{Ge}_{30}$ and $\mathrm{Eu}_{4} \mathrm{Sr}_{4} \mathrm{Ga}_{16} \mathrm{Ge}_{30}$ from a different perspective, we have investigated the MCE of the two specimens and performed MCE-based critical analysis. The magnetic entropy change $\left(\Delta S_{M}\right)$ was calculated from isothermal magnetization (M-H) curves using the Maxwell relation, ${ }^{7}$

$$
\Delta S_{M}=\mu_{0} \int_{0}^{H_{\max }}\left(\frac{\partial M}{\partial T}\right)_{H} d H,
$$

where $M$ is the magnetization, $H$ is the magnetic field, and $T$ is the temperature. The results of the calculated $\Delta S_{M}$ are shown in Figs. 5(a) and 5(b) for $\mathrm{Eu}_{8} \mathrm{Ga}_{16} \mathrm{Ge}_{30}$ and $\mathrm{Eu}_{4} \mathrm{Sr}_{4} \mathrm{Ga}_{16} \mathrm{Ge}_{30}$, respectively. As evident in Fig. 5, an additional shoulder is present at $T_{L} \sim 10 \mathrm{~K}$ for $\mathrm{Eu}_{8} \mathrm{Ga}_{16} \mathrm{Ge}_{30}$, in addition to the expected peak at $T_{C} \sim 35 \mathrm{~K}$. This feature is largely suppressed for $\mathrm{Eu}_{4} \mathrm{Sr}_{4} \mathrm{Ga}_{16} \mathrm{Ge}_{30}$. The occurrence of the broader peak at $13 \mathrm{~K}$ in the $\Delta S_{M}(T)$ curves (see Fig. 5(b)) is presumably a combination of the features at $T_{C} \sim 15 \mathrm{~K}$ and $T_{L} \sim 5 \mathrm{~K}$. A similar result has been reported for the case of a multiple magnetic phase transition system $\mathrm{Nd}_{1.25} \mathrm{Fe}_{11} \mathrm{Ti}^{24}$

In a study of MCE in EuO, the minority phase $\mathrm{Eu}_{3} \mathrm{O}_{4}$ was also detected at $\sim 5 \mathrm{~K} .{ }^{25}$ Another clathrate structure type, $\mathrm{Eu}_{4} \mathrm{Ga}_{8} \mathrm{Ge}_{16}$, has also been reported to undergo antiferromagnetic ordering at $\sim 8 \mathrm{~K} .{ }^{26,27}$ Although no impurity phases were detected in our specimens, in order to investigate the possibility that $T_{L}$ arises from $\mathrm{Eu}_{3} \mathrm{O}_{4}$ or other impurity phases, we conducted systematic structural and MCE analyses on three different $\mathrm{Eu}_{8} \mathrm{Ga}_{16} \mathrm{Ge}_{30}$ specimens. While the XRD patterns reveal only the type-I clathrate phase, MCE data confirmed the presence of $T_{L}$ in all three specimens. This analysis, together with our extensive XRD and EDS analyses, suggests that the presence of $T_{L}$ is an intrinsic property of the $\mathrm{Eu}_{8} \mathrm{Ga}_{16} \mathrm{Ge}_{30}$ type-I clathrate.

Recently, Franco et al. ${ }^{28-32}$ have shown that for SOMT materials the $\Delta S_{M}(T)$ curves measured with different maximum applied fields will collapse onto a universal curve (which is unique for each universality class, regardless of the particular specimen) by normalizing all the $\Delta S_{M}(T)$ curves to their respective peak value $\Delta S_{M}^{p k}$ (i.e., $\Delta \mathrm{S}^{\prime}=\Delta \mathrm{S}_{\mathrm{M}}(\mathrm{T}) / \Delta S_{M}^{p k}$ ) and rescaling the temperature axis above and below $T_{C}$,

$$
\theta \equiv \theta_{1}=\left(T-T_{C}\right) /\left(T_{r}-T_{C}\right),
$$

where $\mathrm{T}_{\mathrm{r}}$ is the reference temperature corresponding to a certain fraction $f$ that fulfils $\Delta S_{M}\left(T_{r}\right) / \Delta S_{M}^{p k}=f$. This choice of $f$ does not affect the actual construction of the universal curve, as it implies only a proportionality constant. When the material deviates from ideal behavior (for example a material with multiple magnetic phases), it is necessary to use two well-separated reference temperatures, $T_{r 1}$ and $T_{r 2}$, to construct the universal curve, ${ }^{28,31}$

$$
\theta \equiv \theta_{2}= \begin{cases}-\left(T-T_{C}\right) /\left(T_{r 1}-T_{C}\right) ; & T \leqslant T_{C} \\ \left(T-T_{C}\right) /\left(T_{r 2}-T_{C}\right) ; & T>T_{C}\end{cases}
$$

In the present study, the reference temperatures $T_{r} 1$ and $T_{r} 2$ have been selected as those corresponding to $0.6 \Delta S_{M}^{p k}$.

Figure 6 shows the universal curves for $\mathrm{Eu}_{8} \mathrm{Ga}_{16} \mathrm{Ge}_{30}$ and $\mathrm{Eu}_{4} \mathrm{Sr}_{4} \mathrm{Ga}_{16} \mathrm{Ge}_{30}$ using Eq. (5) for the case of $\theta_{1}$ and Eq. (6) for the case of $\theta_{2}$, respectively. As clearly indicated in Fig. 6, the overlap of the different curves is reasonable around $T_{C}$, however, not in the $\theta_{1} \leqslant-2$ range $\left(T<T_{L} \sim 10 \mathrm{~K}\right)$, although the two reference temperature method yields a 

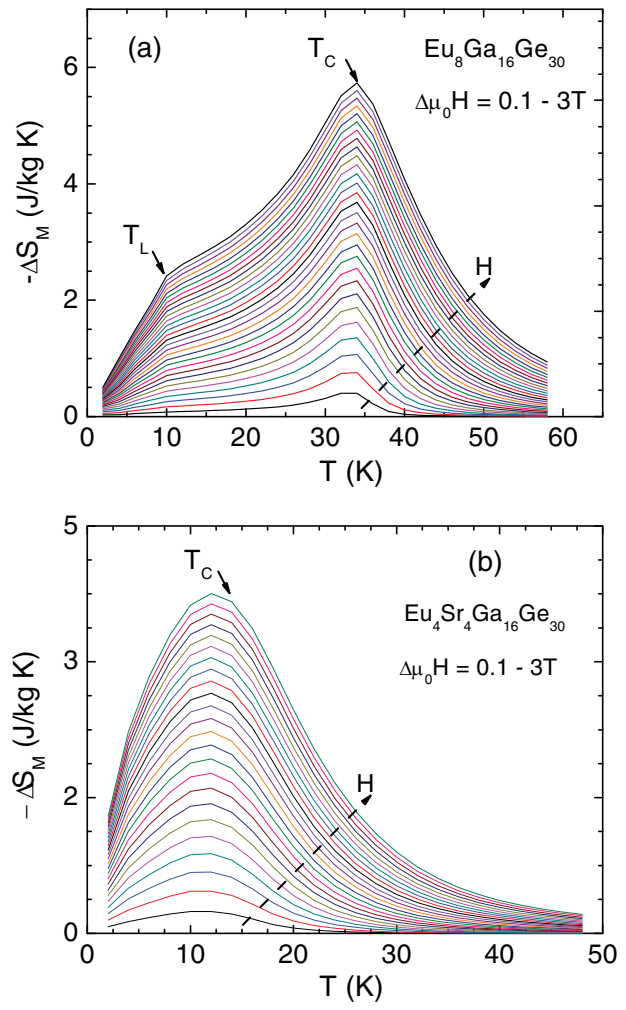

FIG. 5. (Color online) Temperature and magnetic field dependencies of magnetic entropy change $\left(-\Delta \mathrm{S}_{\mathrm{M}}\right)$ for (a) $\mathrm{Eu}_{8} \mathrm{Ga}_{16} \mathrm{Ge}_{30}$ and (b) $\mathrm{Eu}_{4} \mathrm{Sr}_{4} \mathrm{Ga}_{16} \mathrm{Ge}_{30}$. Dashed arrows indicate the direction of the increasing magnetic field.

slightly better overlap for temperatures below $T_{C}$. It has been noted that perfect overlap of the curves is achieved in the entire temperature range for single magnetic phase transition materials $^{28,30}$ when a single reference temperature is used but not for multiple magnetic phase transition materials, ${ }^{29,34}$ although the influence of the second phase transition in the close environment of the $T_{C}$ of the transition under this study
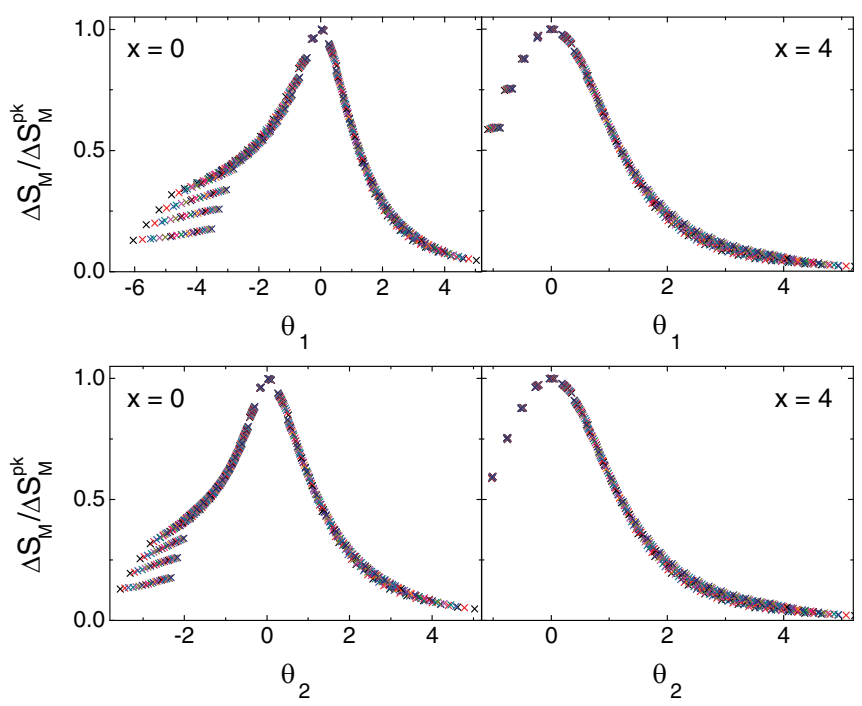

FIG. 6. (Color online) The universal curves for $\mathrm{Eu}_{8} \mathrm{Ga}_{16} \mathrm{Ge}_{30}$ and $\mathrm{Eu}_{4} \mathrm{Sr}_{4} \mathrm{Ga}_{16} \mathrm{Ge}_{30}$ using Eq. (5) in the case of $\theta_{1}$ and Eq. (6) in the case of $\theta_{2}$, respectively.
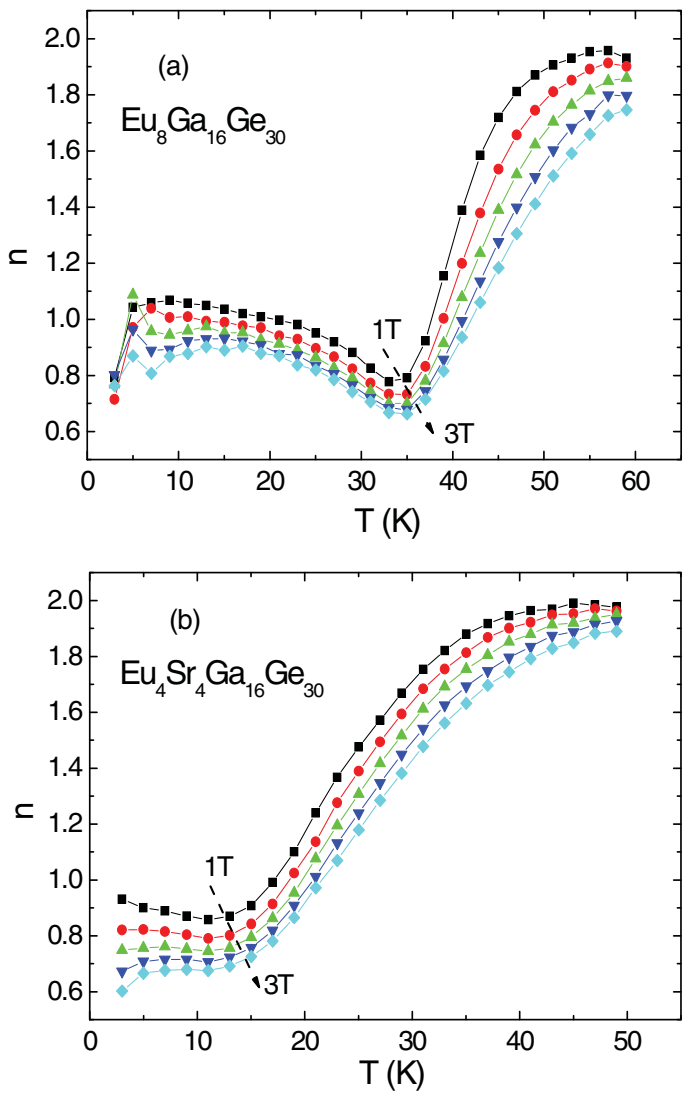

FIG. 7. (Color online) Temperature dependence of the local exponent $n$ for (a) $\mathrm{Eu}_{8} \mathrm{Ga}_{16} \mathrm{Ge}_{30}$ and (b) $\mathrm{Eu}_{4} \mathrm{Sr}_{4} \mathrm{Ga}_{16} \mathrm{Ge}_{30}$ at different magnetic fields from 1 to $3 \mathrm{~T}$ with a field interval of $0.5 \mathrm{~T}$.

can be minimized by using the two reference temperatures. The lack of perfect overlap for $\mathrm{Eu}_{8} \mathrm{Ga}_{16} \mathrm{Ge}_{30}$ at temperatures well below $T_{C}$ is not a failure of the universal curve model but may be an indication of an additional magnetic transition at $T_{L}$ with different field dependencies for this specimen. The larger differences between both procedures (using $\theta_{1}$ or $\theta_{2}$ ) are seen for the $\mathrm{Eu}_{4} \mathrm{Sr}_{4} \mathrm{Ga}_{16} \mathrm{Ge}_{30}$ due to the closer temperatures $T_{C}$ and $T_{L}(\sim 10 \mathrm{~K}$ apart $)$ when compared with $\mathrm{Eu}_{8} \mathrm{Ga}_{16} \mathrm{Ge}_{30}(\sim 25 \mathrm{~K}$ apart). As the contribution to the magnetic entropy change quickly goes to zero when the temperature increases above $T_{L}$, there is little benefit in the analysis on the $\mathrm{Eu}_{8} \mathrm{Ga}_{16} \mathrm{Ge}_{30}$ specimen in using $\theta_{2}$. However, the larger convolution of the peaks associated with $T_{C}$ and $T_{L}$ for the $\mathrm{Sr}$-doped specimen improves the overlap as $\theta_{2}$ is used. The need for using two reference temperatures for the $\mathrm{Sr}$-doped specimen is an indication that $T_{L}$ is not completely suppressed by doping.

It has also been demonstrated that the field dependence of $\Delta S_{M}$ can be expressed as $\Delta S_{M}^{p k} \propto H^{n}$ for some materials. ${ }^{28,30}$ The exponent $n$ can be locally calculated from the logarithmic derivative of the $\Delta S_{M}$ with magnetic field as

$$
n(T, H)=\frac{d \ln \left|\Delta S_{M}\right|}{d \ln H},
$$

giving $n=2$ in the paramagnetic range for $T \gg T_{C}, n=1$ for $T \ll T_{C}$, and

$$
n\left(T=T_{C}\right)=1+1 / \delta(1-1 / \beta)=(1-\alpha) / \Delta
$$


for $T=T_{C} \cdot{ }^{28}$ It has been reported that $n$ depends on the magnetic field and temperature of the material. ${ }^{28-31}$ For the case of single magnetic phase materials, $n$ is field independent at temperature $T_{C}$ or the temperature of the peak entropy change. ${ }^{28,30,33}$ It evolves with magnetic field at any temperature for the case of multiphase materials. ${ }^{24,34,35}$

Figures 7(a) and 7(b) show the temperature and magnetic field dependences of $n$ calculated using Eq. (7) for $\mathrm{Eu}_{8} \mathrm{Ga}_{16} \mathrm{Ge}_{30}$ and $\mathrm{Eu}_{4} \mathrm{Sr}_{4} \mathrm{Ga}_{16} \mathrm{Ge}_{30}$, respectively. For both specimens, $n$ approaches 2 in the paramagnetic range at $T \gg$ $T_{C} \cdot{ }^{28}$ As summarized in Table I, the values of $n$ at $T_{C}$ [denoted as $\left.n\left(T_{C}\right)\right]$ calculated from the $\Delta S_{M}(T, H)$ data $\left[n\left(T_{C}\right)=0.562\right.$ and 0.675 for $\mathrm{Eu}_{8} \mathrm{Ga}_{16} \mathrm{Ge}_{30}$ and $\mathrm{Eu}_{4} \mathrm{Sr}_{4} \mathrm{Ga}_{16} \mathrm{Ge}_{30}$, respectively] are consistent with those calculated from Eq. (8) using the values of $\beta$ and $\delta$ (obtained from the K-F method $\left[n\left(T_{C}\right)=\right.$ 0.545 and 0.667 for $\mathrm{Eu}_{8} \mathrm{Ga}_{16} \mathrm{Ge}_{30}$ and $\mathrm{Eu}_{4} \mathrm{Sr}_{4} \mathrm{Ga}_{16} \mathrm{Ge}_{30}$, respectively]. The agreement between the exponent $n$ obtained from the K-F method and the MCE analysis supports the application of the MCE analysis to determine the critical exponents in the cases when the K-F method cannot be used. ${ }^{31,32}$ In addition, the anomalous magnetic field dependence of $n$ has been noted at $T_{L} \sim 10 \mathrm{~K}$ for $\mathrm{Eu}_{8} \mathrm{Ga}_{16} \mathrm{Ge}_{30}$ [see Fig. 7(a)]. The plateau feature below $T_{C}$ in $n(T)$ for $\mathrm{Eu}_{4} \mathrm{Sr}_{4} \mathrm{Ga}_{16} \mathrm{Ge}_{30}$ [see Fig. 7(b)] can be reconciled with the broader nature of the $\Delta S_{M}(T)$ peak due to the overlap of the peaks associated with $T_{C}$ and $T_{L}$ [see Fig. 5(b)]. The theoretically predicted relationship between the peak entropy change $\left(\Delta S_{M}^{p k}\right)$ and the magnetic field, $\Delta S_{M}^{p k} \propto H^{n}$, has been confirmed for both specimens (see Fig. 8).

From Table I we note that for the $\mathrm{Eu}_{8} \mathrm{Ga}_{16} \mathrm{Ge}_{30}$ type-I clathrate the critical exponent $\beta=0.388$ is close to that predicted from the 3D Heisenberg model $(\beta=0.365)$ with short-range ferromagnetic correlation, while the critical exponent $\gamma=0.956$ is close to that predicted from the mean-field model $(\gamma=1)$. The exponent of this specimen $n\left(T_{C}\right)=0.545$ significantly deviates from that predicted from the mean-field

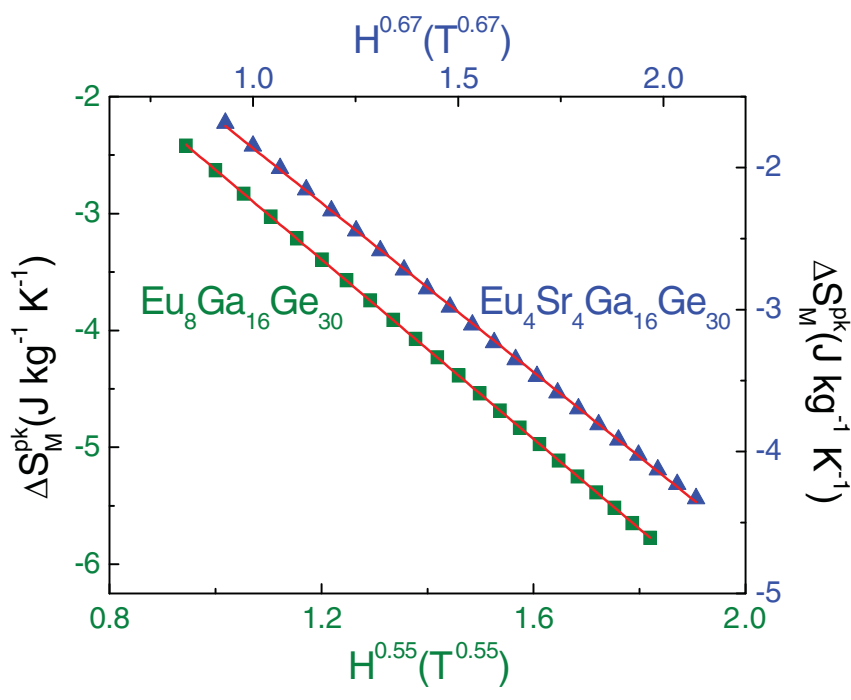

FIG. 8. (Color online) $\Delta S_{M}^{p k}$ vs $H^{n}$ with $n=0.67$ and 0.55 . The linear $\Delta S_{M}^{p k} \propto H^{n}$ relationship at $T_{C}$ for $\mathrm{Eu}_{8} \mathrm{Ga}_{16} \mathrm{Ge}_{30}$ (bottom and left axis) and $\mathrm{Eu}_{4} \mathrm{Sr}_{4} \mathrm{Ga}_{16} \mathrm{Ge}_{30}$ (top and right axis) is shown in the figure. model $\left(n\left(T_{C}\right)=2 / 3\right)$. At first glance, one may infer that the magnetic interaction in the $\mathrm{Eu}_{8} \mathrm{Ga}_{16} \mathrm{Ge}_{30}$ type-I clathrate near $T_{C}$ is of short-range type. However, the ferromagnetism of this material has been proved to be governed by the RKKY mechanism with long-range interaction, ${ }^{4,5,9,10}$ which thus rules out this hypothesis. In the $\mathrm{Eu}_{8} \mathrm{Ga}_{16} \mathrm{Ge}_{30}$ type-I clathrate $\mathrm{Eu}^{2+}$ tunnels between four equivalent sites in the tetrakaidecahedral cages and the tunneling states correspond to the static positional disorder induced by the split site Eu2. ${ }^{11,12}$ It has been shown that in addition to the "rattling" motion, or dynamic disorder, of the $\mathrm{Eu}^{2+}$ ions, the tunneling of $\mathrm{Eu}^{2+}$ ions plays an important role in producing the glasslike low thermal conductivity in this material. ${ }^{4,5,12}$ While the thermal conductivity of both $\mathrm{Eu}_{8} \mathrm{Ga}_{16} \mathrm{Ge}_{30}$ and $\mathrm{Sr}_{8} \mathrm{Ga}_{16} \mathrm{Ge}_{30}$ was not affected by an applied magnetic field $(\sim 8 \mathrm{~T})$, this magnetic field significantly suppressed the spin-disorder scattering, which consequently increased the electrical conductivity of $\mathrm{Eu}_{8} \mathrm{Ga}_{16} \mathrm{Ge}_{30}$, leading to the "negative" magnetoresistance effect (the $10 \%$ decrease in resistance with an applied field of $8 \mathrm{~T}$ ) around its Curie temperature $\left(T_{C} \sim 35 \mathrm{~K}\right) .{ }^{4}$ In the present study, we show that the tunneling of $\mathrm{Eu}^{2+}$ between four equivalent sites in the tetrakaidecahedral cage may be the reason for the ferromagnetic ordering and MCE in the type-I clathrate materials. It has been noted that the tunneling of $\mathrm{Eu}^{2+}$ ions can lead to the coexistence of competing multiple ferromagnetic states or the modulated ferromagnetic structure below $T_{C} \cdot{ }^{2,14}$ We believe that the formation of the modulated ferromagnetic structure causes the critical exponents $\beta$ and $n\left(T_{C}\right)$ of the $\mathrm{Eu}_{8} \mathrm{Ga}_{16} \mathrm{Ge}_{30}$ type-I clathrate to deviate from those predicted by the mean-field theory. This is plausible if one considers the significant difference in the critical exponents between the $\mathrm{Eu}_{8} \mathrm{Ga}_{16} \mathrm{Ge}_{30}$ type-I clathrate and the $\mathrm{Eu}_{8} \mathrm{Ga}_{16} \mathrm{Ge}_{30}$ type-VIII clathrate (see Table I). In contrast to the case of the $\mathrm{Eu}_{8} \mathrm{Ga}_{16} \mathrm{Ge}_{30}$ type-I clathrate, tunneling of $\mathrm{Eu}^{2+}$ ions does not occur in the $\mathrm{Eu}_{8} \mathrm{Ga}_{16} \mathrm{Ge}_{30}$ type-VIII clathrate, while the critical exponents $\beta=0.470$ and $\gamma=0.964$ of type-VIII $\mathrm{Eu}_{8} \mathrm{Ga}_{16} \mathrm{Ge}_{30}$ are close to those predicted from the mean-field model ( $\beta=0.5$ and $\gamma=1$ ) with long-range ferromagnetic correlations. ${ }^{7}$ This implies that the tunneling of $\mathrm{Eu}^{2+}$ between the four equivalent sites in the tetrakaidecahedral cage tends to prevent the occurrence of a long-range ferromagnetic ordering in the type-I clathrate material. This tunneling could cause the magnitude of the jump in $C_{p}$ at $T_{C}$ to significantly differ from the value expected for a uniform ferromagnet with $S=7 / 2$ from the mean-field theory. ${ }^{4,5,14}$

From a magnetic cooling application perspective, the refrigerant capacity (RC) is considered as one of the most important factors for assessing the usefulness of a magnetic refrigerant material. The RC depends not only on the magnitude of $\Delta S_{M}$ but also on its temperature dependence (e.g., the full width at half maximum of the $\Delta S_{M}(T)$ peak). While the $\mathrm{Eu}_{8} \mathrm{Ga}_{16} \mathrm{Ge}_{30}$ type-VIII clathrate shows a relatively narrow $\Delta S_{M}(T)$ curve resulting in the moderate $\mathrm{RC}(\sim 159 \mathrm{~J} / \mathrm{kg}$ at $3 \mathrm{~T}),{ }^{7}$ the tunneling of $\mathrm{Eu}^{2+}$ ions in the $\mathrm{Eu}_{8} \mathrm{Ga}_{16} \mathrm{Ge}_{30}$ type-I clathrate likely leads to the broadened $\Delta S_{M}(\mathrm{~T})$ curve thus enhancing the $\mathrm{RC}$ in this material $(\sim 174 \mathrm{~J} / \mathrm{kg}$ at $3 \mathrm{~T})$. This finding may provide a route for improving the cooling efficiency in type-I clathrate materials for advanced magnetic refrigeration applications. More importantly, these results provide a better understanding of the fundamental difference 
in the magnetic and magnetocaloric properties between the type-I and type-VIII clathrates.

\section{CONCLUSIONS}

The nature of the ferromagnetic phase transitions and critical exponents in $\mathrm{Eu}_{8} \mathrm{Ga}_{16} \mathrm{Ge}_{30}$ and $\mathrm{Eu}_{4} \mathrm{Sr}_{4} \mathrm{Ga}_{16} \mathrm{Ge}_{30}$ has been studied systematically. $\mathrm{Eu}_{8} \mathrm{Ga}_{16} \mathrm{Ge}_{30}$ undergoes a SOMT at $T_{C} \sim 35 \mathrm{~K}$, as a result of the magnetic interaction between the $\mathrm{Eu}^{2+}$ ions at the $\mathrm{Eu} 2$ sites, followed by a secondary ferromagnetic transition at $T_{L} \sim 10 \mathrm{~K}$, resulting from the magnetic interaction between the $\mathrm{Eu}^{2+}$ ions at the Eu1 and Eu2 sites. $\mathrm{Eu}_{4} \mathrm{Sr}_{4} \mathrm{Ga}_{16} \mathrm{Ge}_{30}$ retains the SOMT but largely reduces the transition temperatures $T_{C} \sim 15 \mathrm{~K}$ and
$T_{L} \sim 5 \mathrm{~K}$. As shown in Table I, the substitution of $\mathrm{Sr}^{2+}$ for $\mathrm{Eu}^{2+}$ in $\mathrm{Eu}_{4} \mathrm{Sr}_{4} \mathrm{Ga}_{16} \mathrm{Ge}_{30}$ increases the critical exponents $\beta=0.521$ and $n\left(T_{C}\right)=0.667$ and brings them close to those predicted from the mean-field model ( $\beta=0.5$ and $n\left(T_{C}\right)$ $=2 / 3$ ). The critical exponents derived from the K-F and MCE methods suggest that the tunneling of $\mathrm{Eu}^{2+}$ between the four equivalent sites in the tetrakaidecahedral cage favors the occurrence of a short-range ferromagnetic ordering in $\mathrm{Eu}_{8} \mathrm{Ga}_{16} \mathrm{Ge}_{30}$.

\section{ACKNOWLEDGMENTS}

This work is supported by the Army Research Office through Grant No. W911NF-08-1-0276. *gnolas@usf.edu

${ }^{\dagger}$ Corresponding author: sharihar@usf.edu

${ }^{1}$ G. S. Nolas, G. A. Slack, and S. B. Schujman, in Semiconductors and Semimetals, edited by T. M. Tritt (Academic Press, New York, 2001), vol. 69, p. 255.

${ }^{2}$ G. S. Nolas, J. L. Cohn, G. A. Slack, and S. B. Schujman, Appl. Phys. Lett. 73, 178 (1998).

${ }^{3}$ Y. Sasaki, K. Kishimoto, T. Koyanagi, H. Asada, and K. Akai, J. Appl. Phys. 105, 073702 (2009).

${ }^{4}$ B. C. Sales, B. C. Chakoumakos, R. Jin, J. R. Thompson, and D. Mandrus, Phys. Rev. B 63, 245113 (2001).

${ }^{5}$ S. Paschen, W. Carrillo-Cabrera, A. Bentien, V. H. Tran, M. Baenitz, Y. Grin, and F. Steglich, Phys. Rev. B 64, 214404 (2001).

${ }^{6}$ G. T. Woods, J. Martin, M. Beekman, R. P. Hermann, F. Grandjean, V. Keppens, O. Leupold, Gary J. Long, and G. S. Nolas, Phys. Rev. B 73, 174403 (2006).

${ }^{7}$ M. H. Phan, G. T. Woods, A. Chaturvedi, S. Stefanoski, G. S. Nolas, and H. Srikanth, Appl. Phys. Lett. 93, 252505 (2008).

${ }^{8}$ M. H. Phan, V. Franco, A. Chaturvedi, S. Stefanoski, G. T. Woods, G. S. Nolas, and H. Srikanth, J. Appl. Phys. 107, 09A910 (2010).

${ }^{9}$ V. Pacheco, A. Bentien, W. Carrillo-Cabrera, S. Paschen, F. Steglich, and Y. Grin, Phys. Rev. B 71, 165205 (2005).

${ }^{10}$ A. Bentien, V. Pacheco, S. Paschen, Y. Grin, and F. Steglich, Phys. Rev. B 71, 165206 (2005).

${ }^{11}$ R. P. Hermann, V. Keppens, P. Bonville, G. S. Nolas, F. Grandjean, G. J. Long, H. M. Christen, B. C. Chakoumakos, B. C. Sales, and D. Mandrus, Phys. Rev. Lett. 97, 017401 (2006).

${ }^{12}$ B. C. Chakoumakos, B. C. Sales, and D. G. Mandrus, J. Alloys Compd. 322, 127 (2001).

${ }^{13} \mathrm{H}$. Tomono, H. Eguchi, and K. Tsumuraya, J. Phys. Condens. Matter 20, 385209 (2008).

${ }^{14}$ T. Onimaru, S. Yamamoto, M. A. Avila, K. Suekuni, T. Takabatake, J. Phys. Conf. Ser. 200, 022044 (2010).

${ }^{15}$ J. L. Cohn, G. S. Nolas, V. Fessatidis, T. H. Metcalf, and G. A. Slack, Phys. Rev. Lett. 82, 779 (1999).

${ }^{16}$ Y. Zang, P. L. Lee, G. S. Nolas, and P. Wilkinson, Appl. Phys. Lett. 80, 2931 (2002).

${ }^{17}$ G. S. Nolas, T.J.R. Weakley, J. L. Cohn, and R. Sharma, Phys. Rev. B 61, 3845 (2000).
${ }^{18}$ S. K. Banerjee, Phys. Rev. Lett. 12, 16 (1964).

${ }^{19}$ J. Mira, J. Rivas, F. Rivadulla, C. Vázquez-Vázquez, and M. A. Lopez-Quintela, Phys. Rev. B 60, 2998 (1999).

${ }^{20}$ M. H. Phan, S. C. Yu, N. H. Hur, and Y. H. Jeong, J. Appl. Phys. 96, 1154 (2004).

${ }^{21}$ J. S. Kouvel and M. E. Fisher, Phys. Rev. 136, A1626 (1964).

${ }^{22}$ B. Widom, J. Chem. Phys. 43, 3898 (1965).

${ }^{23} \mathrm{H}$. Eugene Stanley, Introduction to Phase Tansitions and Critical Phenomena (Oxford University Press, New York, 1971).

${ }^{24}$ R. Caballero-Flores, V. Franco, A. Conde, Q. Y. Dong, and H. W. Zhang, J. Magn. Magn. Mater. 322, 804 (2010).

${ }^{25}$ K. Ahn, A. O. Pecharsky, K. A. Gschneidner, and V. K. Pecharsky, J. Appl. Phys. 97, 063901 (2004).

${ }^{26}$ M. Christensen, J. D. Bryan, H. Birkedal, G. D. Stucky, B. Lebech, and B. B. Iversen, Phys. Rev. B 68, 174428 (2003).

${ }^{27}$ J. Daniel Bryan, H. Trill, H. Birkedal, M. Christensen, Vojislav I. Srdanov, H. Eckert, Bo B. Iversen, and Galen D. Stucky, Phys. Rev. B 68, 174429 (2003).

${ }^{28}$ V. Franco, J. S. Blazquez, and A. Conde, Appl. Phys. Lett. 89, 222512 (2006).

${ }^{29}$ V. Franco, A. Conde, V. K. Pecharsky, and K. A. Gschneidner Jr., Europhys. Lett. 79, 47009 (2007).

${ }^{30}$ V. Franco, A. Conde, J. M. Romero-Enrique, and J. S. Blazquez, J. Phys. Condens. Matter 20, 285207 (2008).

${ }^{31}$ V. Franco, A. Conde, V. Provenzano, and R. D. Shull, J. Magn. Magn. Mater. 322, 218 (2010).

${ }^{32}$ V. Franco, A. Conde, D. Sidhaye, B. L. V. Prasad, P. Poddar, S. Srinath, M. H. Phan, and H. Srikanth, J. Appl. Phys. 107, 09A902 (2010).

${ }^{33}$ V. Franco, A. Conde, M. D. Kuz'min, and J. M. Romero Enrique, J. Appl. Phys. 105, 07A917 (2009).

${ }^{34}$ V. Franco, R. Cabalero-Flores, A. Conde, Q. Y. Dong, and H. W. Zhang, J. Magn. Magn. Mater. 321, 1115 (2009).

${ }^{35}$ Q. Y. Dong, H. W. Zhang, J. R. Sun, B. G. Shen, and V. Franco, J. Appl. Phys. 103, 116101 (2008).

${ }^{36}$ M. Seeger, S. N. Kaul, H. Kronmuller, and R. Reisser, Phys. Rev. B 51, 12585 (1995). 